\section{Barbara Wurster}

zu ihrem neuen Amt (ab 1. Juni 2010) als erste Programmdirektorin (Head of Operations Unit) am neuen Europäischen Gleichstellungsinstitut in Vilnius - EIGE. Das Europäische Gleichstellungsinstitut ist eine Einrichtung der Europäischen Union, die speziell auf Stärkung und Förderung von Geschlechter-Gleichstellungsfragen abzielt. Das Institut will vergleichbare, verlässliche Daten auf europäischer Ebene sammeln, analysieren und auch durch Erfahrungsaustausch dazu beitragen, die Integration von Gleichstellungsaspekten in allen Politikbereichen der Gemeinschaft zu fördern. Barbara Wurster war von 2001 bis 2007 Vizepräsidentin des djb und Vorsitzende der Regionalgruppe Bonn von 1997 bis 2007.

\section{Geburtstage}

(Januar bis März 2010)

\section{Jahre}

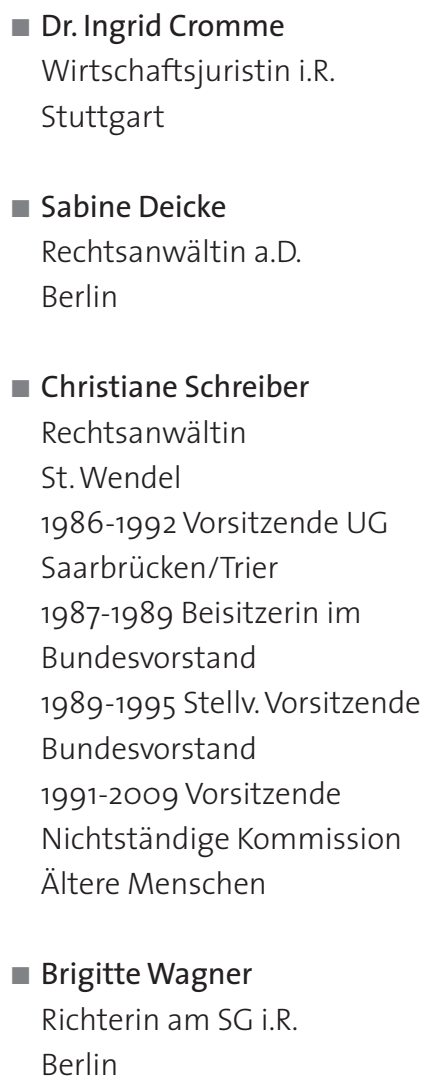

75 Jahre

- Barbara Breiholdt

Rechtsanwältin

Hamburg

Vorstandsmitglied

LV Hamburg 1990-1996

- Ingrid Metzeler

Richterin am AG i.R.

Ratingen

- Ingeburg Michel

Vors. Richterin am LG i.R.

Heidelberg

\section{Jahre}

- Dr. Gesine van Randenborgh Landesverwaltungsdirektorin i.R.

Köln

\section{Jahre \\ - Vera Movsessian Rechtsanwältin und Notarin Berlin}

\section{Jahre}

- Annette Schücking-Homeyer Richterin i.R.

Lünen

Gründungs- und

Ehrenmitglied des djb

\title{
Annette Schücking-Homeyer zum 90. Geburtstag
}

\author{
Dr. Marion Röwekamp \\ Kennedy Fellow am Minda de Gunzburg Center for European \\ Studies, Harvard University
}

Annette Schücking wurde am 1. März 1920 in Dortmund als Tochter eines Rechtsanwalts geboren, dem 1933 wegen „kommunistischer Betätigung “ (der Vater war SPD-Mitglied und Demokrat) die Zulassung entzogen wurde. Der Vater tauchte unter, die Mutter bemühte sich um den Lebensunterhalt. Sie wurde Bäuerin, um die Familie zu ernähren, die dieswegen nach Sassenberg bei Warendorf aufs Land zog. Nach dem Abitur beschloss Annette Schücking, Jura zu studieren. Anlass war die Information, dass der SPD-Reichstagsabgeordnete
Ernst Heilmann, der im Juni 1933 verhaftet worden war, im Konzentrationslager Buchenwald - wo er im April 1940 ermordet wurde - in einer Hundhütte leben musste. Für die Familie war es selbstverständlich, dass auch die Töchter einen Beruf ergreifen und sich nicht von Ehemännern abhängig machen sollten. Die Aussichten für Juristinnen waren 1938 düster, doch das hielt sie nicht davon ab, nach Absolvierung des Arbeitsdienstes zum Wintersemester 1938/39 zunächst in Münster, dann in München und Göttingen Jura zu studieren. 1941 bestand sie das Referendarexamen, wurde jedoch zunächst nicht zur Referendarin ernannt. Sie bewarb sich daher beim Roten Kreuz und verbrachte die Zeit bis Anfang 1943 als DRK-Helferin in der Ukraine. Nach ihrer Rückkehr be- 
gann sie am Amtsgericht Warendorf mit dem Referendariat. Das Assessorexamen legte sie, bedingt durch Krieg und Kriegsende, erst 1947 ab. Anschließend war sie zunächst als Rechtsanwältin, später als Richterin tätig. 1952 wurde sie zur Amtsgerichtsrätin beim Amtsgericht in Duisburg ernannt. 1954 wurde sie Richterin am Sozialgericht Düsseldorf und befasste sich mit Fragen der Renten- und Krankenversicherung. Nach der Geburt ihrer Kinder wurde sie antragsgemäß an das Sozialgericht Detmold versetzt. Dort blieb sie bis zum Ruhestand 1983. 1979 gründete sie zusammen mit ihrer Schwester das Frauenhaus in Warendorf. Annette Schücking-Homeyer ist Gründungs- und Ehrenmitglied des djb.

\section{Interview mit Annette Schücking-Homeyer am 22. April 2010 zur Gründung des Juristinnenbunds 1948 in Dortmund}

Annette Schücking-Homeyer, das einzig noch lebende Gründungsmitglied des djb, feierte am 1. März dieses Jahres ihren 90. Geburtstag. Obwohl die ehemalige Richterin am Sozialgericht nach der Gründung des djb in keiner Kommission tätig wurde, beobachtete sie über Jahre mit klarem Blick das Vereinsgeschehen und ergriff das Wort, wenn sie den eingeschlagenen Weg für problematisch hielt. Über Briefe an die Geschäftsstelle präsent, nahm sie häufig mahnend, den emanzipatorischen Kurs des djb mit mehr Vehemenz zu vertreten, aber auch lobend am Schicksal des djb teil. Diese Haltung nahm Annette Schücking-Homeyer von Anfang an ein, wie zwei wiedergefundene Briefe an ihre Mutter aus der Gründungszeit des Vereins 1948 zeigen.

Den runden Geburtstag von Annette Schücking-Homeyer nahm ich am 22. April 2010 zum Anlass, mit ihr über ihre Erinnerungen an die Gründung des Verbands zu sprechen.

Annette Schücking ging in Dortmund und in Sassenberg bzw. Warendorf zur Schule. Nachdem sie das Assessorexamen bestanden hatte, musste sie nach den geltenden Ausbildungsvorschriften noch ein Jahr bei einem Anwalt als sogenannte Anwaltsassessorin tätig werden, bevor sie sich selbst als Anwalt niederlassen durfte. Annette Schücking suchte nach einer derartigen Ausbildungsstation und erhielt, obwohl ihr Vater vor seinem Berufsverbot 1933 ein einflussreicher Rechtsanwalt in Dortmund gewesen war, von den männlichen Juristen unter fadenscheinigen Gründen nur Absagen. „Wenn man richtig in Not ist, “ schrieb sie deshalb schließlich an ihre Mutter, „soll man zu einer Frau, insbesondere zu einer Kollegin gehen. “ Sie wandte sich also an die einzige Anwältin in Dortmund, Hildegard Gethmann, die in der Saarbrückerstraße ihre Kanzlei hatte. „Dieses Frl. Gethmann ist blond,“ schrieb Schücking, „hat etwas von einer Tigerin, sie scheint das Leben gründlich zu kennen. Ich komme mir ihr gegenüber schrecklich ,brav' vor, unangenehm brav. Bei ihr riecht es förmlich nach Bert Brecht." Hildegard Gethmann war ein burschikoser Typ; um sich Strafmandate zu besorgen, ging sie in Kneipen, um dort mit den Leuten zu trinken. Bei dem ersten Besuch bei Gethmann besprachen die beiden Juristinnen „... bei Kaffee und unzähligen Zigaretten (sie ist eine starke Raucherin und raucht die Zigaretten an einer Art Zange, die sie in der Hand hält) [...]

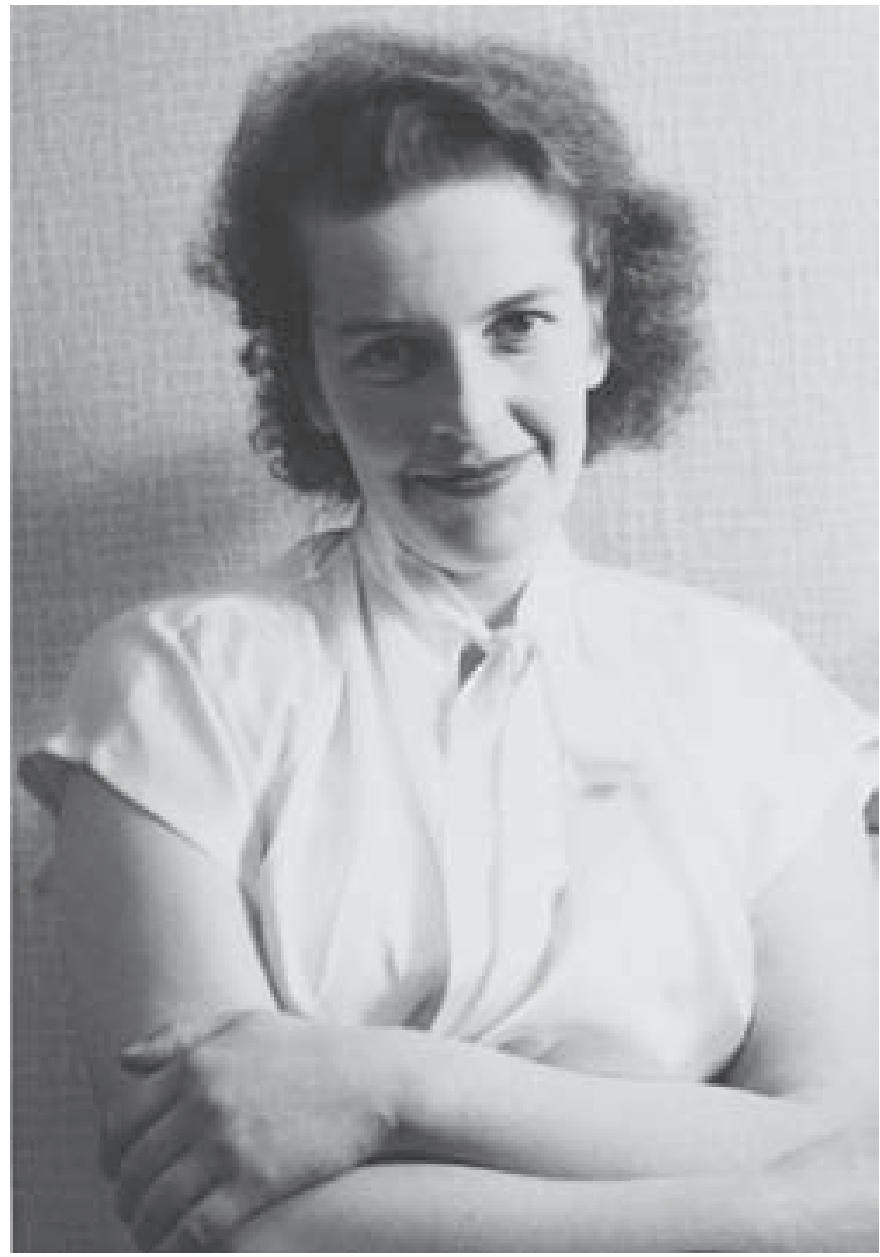

$\Delta$ Annette Schücking-Homeyer (Foto: privat).

den Fall nur kurz. Als dann ein Anwalt aus Hamm hereinbrach, sich hinzusetzte und ich sofort ohne ein Wort zu sagen, eine Tasse aus der danebenliegenden Küche holte, war ich angenommen. “ Der Job bei Frau Gethmann beinhaltete allerdings kein Gehalt. Die Kanzlei Gethmann lief gut, jedoch musste sie die zwei Büroangestellten sowie einige Verwandte mitfinanzieren. Annette Schücking sollte halbtags tätig werden und auch Termine übernehmen, gleichzeitig hatte sie eine halbe Stelle im Stadtarchiv. Dort musste sie die Prozesse der Stadt vorbereiten und Schriftsätze schreiben. Bei dem zukünftigen Dienstvorgesetzten hatte bereits Hildegard Gethmann ihre Ausbildung gemacht und nach einigem Zögern warnte sie ihre junge Kollegin: „M. war ihr gegenüber, wie er es allen Juristinnen gegenüber sein soll, sehr zärtlich, als sie dort arbeitete. Er muss wie P. etwas sein in seinem Gehabe und versucht gerne zu knutschen. Frl. Gethmann musste in ihrer Abwehr dabei einmal einen Spiegel zerschlagen“, schrieb Annette Schücking an ihre Mutter. „Hoffentlich verletzte ich mir nächstens dabei die Schlagadern nicht. Ich werde etwas Jiu-Jitsu lernen müssen. “

Eine der anderen Anwältinnen, die Annette Schücking bei Hildegard Gethmann kennenlernte und ihrer Mutter beschrieb, war Dr. Ruth Rogalski: „(Ostpreussin, hat sich als Bardame das Studium verdient, schwarz, schlank, Mann ist Rechtsanwalt, aber noch nicht zugelassen. Eine 10-jährige Tochter, etwa 45 wie Frl. Gethmann). Sie hat sich auf dersel- 


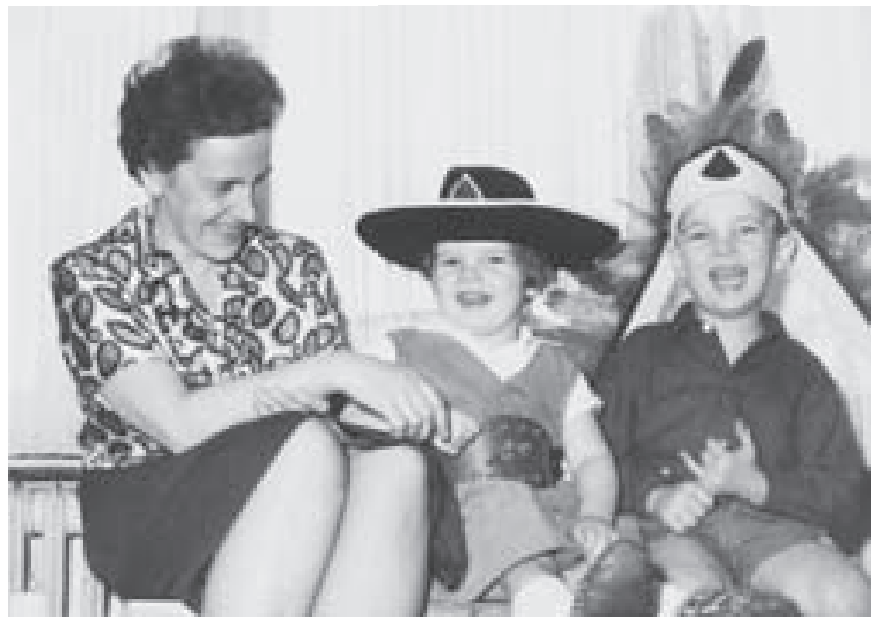

ben Strasse jetzt sechs Zimmer für Büro und Wohnung ausgebaut." Sowohl Gethmann als auch Rogalski waren nach Einschätzung Schückings „stark „Antinazis“ gewesen. Ich glaube, es gibt überhaupt keine Juristin, die in der Partei war.“

Dies entspricht zwar nicht ganz den Tatsachen, aber tatsächlich waren die Juristinnen, die in der Zeit von 1933 bis 1945 wesentlich von der Berufsausübung ausgeschlossen waren, weniger politisch involviert als ihre männlichen Kollegen und traten deshalb, von den Militärregierungen gefördert, nach 1945 vielfach in der Öffentlichkeit auf. Die Beobachtung, dass auch in Dortmund einige Juristinnen vor den Gerichten tätig waren, führte wohl zu der Idee Annette Schückings, einen Juristinnenverein zu gründen. „Mein Vorschlag zu dieser Gründung fiel auf einen wohlgedüngten Boden,“ schrieb sie am 13. August 1948, „Samstag, also morgen in 14 Tagen ist schon bei ihr die erste Tagung der Juristinnen von Dortmund (das sind 6 oder 7). Eigentlich sollte es schon in 8 Tagen sein, aber das geht nicht, da Helmuts Mutter heiratet! “ [Anm. d. Red. Helmut ist Annette Schückings Ehemann.] Also fand die Gründungssitzung der „Vereinigung weiblicher Juristen und Volkswirte e.V.“ eine Woche später, am 28. August 1948, im Büro von Hildegard Gethmann statt. Neben den drei bereits genannten Juristinnen waren auch die Assessorin Luise Purps, die Anwaltsassessorin Alma Schmidt-Perchner, die Rechtsanwältin Elisabeth Späth-Uden sowie die Regierungsrätin Anna Schlieper anwesend. Weil auch die spätere FDPLandtagsabgeordnete Liselotte Funcke mitarbeiten wollte, erweiterten die Juristinnen den Juristinnenverein um die Formulierung „Volkswirtinnen“.

Unklar war in der ersten Sitzung die Zweckbestimmung der Vereinigung. Uneinigkeit gab es vor allem hinsichtlich der Formulierung, dass „die Vereinigung es sich auch zum Ziel gesetzt hat, den Einfluss der Frau in Gesetzgebung, Rechtsprechung, Verwaltung, allen öffentlichen Betrieben, den Wirtschaftskörpern u.s.w. zu verstärken“, wie Schücking schrieb. „Bei der Gründung stemmten sich alle dagegen, dies in die ,Zweckbestimmung' mit hineinzunehmen, um den Männern nicht zu offen den Kampf anzusagen." Darüber hinaus und einstimmig hatte die Vereinigung den Zweck, die beruflichen und wissenschaftlichen Interessen seiner Mitglieder zu för-
4 Die Richterin mit ihren beiden Kindern Anfang der 6oer Jahre (Foto: privat).

dern. Jede Frau, die Rechtswissenschaft oder Volkswirtschaft studierte, war willkommen. Annette Schücking berichtete noch, dass sie zu einem Bekannten ging, um zu erreichen, dass „dass eine Meldung über die Gründung als kurze Nachricht durch alle deutschen Zeitungen hier im Westen geht“. „Die Stadt habe ich dafür eingespannt, dass sie uns unsere Rundschreiben umsonst hektographiert. Ich freue mich schon, was wir Frauen da plötzlich zusammenbekommen. Übrigens kann man jetzt Roben bekommen.“

Diese auch am Alltag orientierten Erinnerungen Annette Schückings machen deutlich, wie knapp finanzielle Mittel so kurz nach der Währungsreform waren. Viele notwendige Dinge mussten privat organisiert werden. Wie rechtspolitisch bewegt die Zeit auf der anderen Seite war und wie sehr die neue Juristinnenvereinigung bereits 1948 mit Musterprozessen Gleichberechtigung erkämpfen wollte, zeigt sich an der Geschichte der Rechtsanwältin Rogalski. Hier berichtet Annette Schücking: „Zur Zeit kämpft sie um die Zulassung ihres Mannes. Diese Schurken haben aus Versehen sie bedingungslos zugelassen, aber nicht ihren Mann, nun hat ihr Mann Schwierigkeiten. Man will sie erpressen, ihre Zulassung ihrem Manne „abzutreten“. Frau Rogalski wird sich, wenn ihr Mann die Zulassung jetzt nicht bekommt, hier in Deutschland scheiden lassen. Das wird ein schöner Prozess sein. Sie wird die Schuld durch die Erklärung übernehmen, dass ihr Beruf ihr mehr wert sei als ihr Mann, und sie nicht auf ihren Beruf verzichte! Frl. Gethmann will dann dieses Urteil in einem gehörigen Pressefeldzug in die Öffentlichkeit ziehen."

Annette Schücking ging bald darauf aus Dortmund weg, weil sie als selbständige Rechtsanwältin ihren Lebensunterhalt nicht bestreiten konnte. „Aber das war nicht einfach “, berichtete sie über diese Zeit, „Männer gingen damals nicht unbedingt zu weiblichen Rechtsanwälten“. Sie bekam eine Stelle beim Oberversicherungsamt in Münster und ab 1952 als Richterin am Strafgericht in Duisburg. Die in Dortmund gegründete Juristinnenvereinigung gewann sehr schnell neue Mitglieder, neue Ortsgruppen wurden gegründet, 1950 wurde sie in Rom in die FIDA aufgenommen und war schon in den ersten Jahren nach ihrer Gründung rechtspolitisch äußerst präsent. 